\title{
Evolution of Quantum Fluctuations Near the Quantum Critical Point of the Transverse Field Ising Chain System $\mathrm{CoNb}_{2} \mathrm{O}_{6}$
}

\author{
A. W. Kinross, ${ }^{1}$ M. Fu, ${ }^{1}$ T. J. Munsie, ${ }^{1}$ H. A. Dabkowska, ${ }^{2}$ G. M. Luke, ${ }^{1,3}$ Subir Sachdev, ${ }^{4}$ and T. Imai ${ }^{1,3^{*}}$ \\ ${ }^{1}$ Department of Physics and Astronomy, McMaster University, Hamilton L8S4M1, Canada \\ ${ }^{2}$ Brockhouse Institute for Materials Research, McMaster University, Hamilton L8S4M1, Canada \\ ${ }^{3}$ Canadian Institute for Advanced Research, Toronto, Ontario M5G1Z8, Canada \\ ${ }^{4}$ Department of Physics, Harvard University, Cambridge, Massachusetts 02138, USA
}

(Received 21 March 2014; published 14 July 2014)

\begin{abstract}
The transverse field Ising chain model is ideally suited for testing the fundamental ideas of quantum phase transitions because its well-known $T=0$ ground state can be extrapolated to finite temperatures. Nonetheless, the lack of appropriate model materials hindered the past effort to test the theoretical predictions. Here, we map the evolution of quantum fluctuations in the transverse field Ising chain based on nuclear magnetic resonance measurements of $\mathrm{CoNb}_{2} \mathrm{O}_{6}$, and we demonstrate the finite-temperature effects on quantum criticality for the first time. From the temperature dependence of the ${ }^{93} \mathrm{Nb}$ longitudinal relaxation rate $1 / T_{1}$, we identify the renormalized classical, quantum critical, and quantum disordered scaling regimes in the temperature $(T)$ vs transverse magnetic field $\left(h_{\perp}\right)$ phase diagram. Precisely at the critical field $h_{\perp}^{c}=5.25 \pm 0.15 \mathrm{~T}$, we observe a power-law behavior, $1 / T_{1} \sim T^{-3 / 4}$, as predicted by quantum critical scaling. Our parameter-free comparison between the data and theory reveals that quantum fluctuations persist up to as high as $T \sim 0.4 J$, where the intrachain exchange interaction $J$ is the only energy scale of the problem.
\end{abstract}

DOI: 10.1103/PhysRevX.4.031008

Subject Areas: Condensed Matter Physics, Magnetism, Strongly Correlated Materials

\section{INTRODUCTION}

The concept of $T=0$ quantum phase transitions has emerged as an overarching theme in strongly correlated electron physics [1-9]. The nature of quantum fluctuations near the quantum critical point, however, remains enigmatic [10]. How well does the quantum criticality account for finite-temperature properties? How high in temperature does the effect of the quantum critical point persist $[10,11]$ ? Do quantum fluctuations remain strong enough at elevated temperatures to account for the mechanism of exotic superconductivity in copper oxides, iron pnictides, and heavy-fermion systems? The dearth of appropriate model materials for rigorously solvable Hamiltonians has not permitted experimentalists to address these fundamental questions concretely, even for the transverse field Ising chain (TFIC) [12], a celebrated textbook example of quantum criticality [6]. Very recently, the Ising chain material $\mathrm{CoNb}_{2} \mathrm{O}_{6}[13-19]$ was proposed as an ideal model system of the TFIC based on neutron scattering measurements in transverse magnetic fields [20], paving a new

*imai@mcmaster.ca

Published by the American Physical Society under the terms of the Creative Commons Attribution 3.0 License. Further distribution of this work must maintain attribution to the author(s) and the published article's title, journal citation, and DOI. avenue to investigate the finite-temperature effects on quantum fluctuations in the vicinity of a quantum critical point (QCP).

The TFIC Hamiltonian is deceptively simple [6,12]:

$$
H=-J \sum_{i}\left(\sigma_{i}^{z} \sigma_{i+1}^{z}+g \sigma_{i}^{x}\right),
$$

where $J$ ( $>0$ for ferromagnetic Ising chains in $\mathrm{CoNb}_{2} \mathrm{O}_{6}$ ) represents the nearest-neighbor spin-spin exchange interaction, $\sigma_{i}^{z(x)}$ is the $z(x)$ component of the Pauli matrix at the $i$ th site, and the dimensionless coupling constant $g$ is related to the transverse magnetic field $h_{\perp}$ applied along the $x$ axis as $g=h_{\perp} / h_{\perp}^{c}$, where $h_{\perp}^{c}$ is the critical field $\left(h_{\perp}^{c}=5.25 \pm 0.15\right.$ Tesla in $\mathrm{CoNb}_{2} \mathrm{O}_{6}$, as shown below). Since $\sigma_{i}^{z}$ and $\sigma_{i}^{x}$ do not commute, the classical Ising Hamiltonian for $g=0$ becomes the quantum TFIC Hamiltonian for $g>0$. The QCP is located at $g=1$, where the applied field is tuned precisely at $h_{\perp}^{c}$; a magnetic field greater than $h_{\perp}^{c}$ coerces the magnetic moments along its direction and transforms the $T=0$ ferromagnetic ground state to a paramagnetic state. See Fig. 1 for the generic theoretical phase diagram of the TFIC [6,21]. In spite of its apparent simplicity, the TFIC served as the foundational model for quantum Monte Carlo simulations [22], and it continues to attract attention in quantum information theory [23]. 


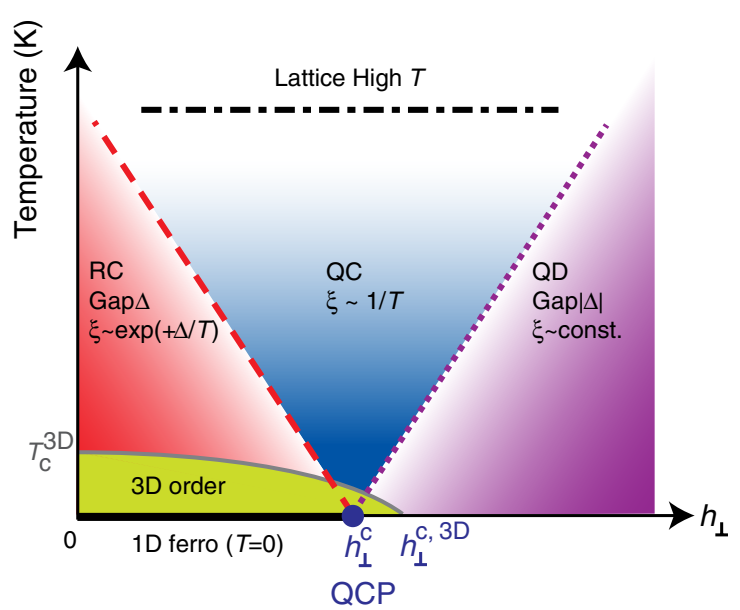

FIG. 1. A generic $T-h_{\perp}$ phase diagram of the TFIC encompasses three scaling regimes with distinct behaviors of the spinspin correlation length $\xi$ : RC $\left[g<1\right.$, hence $h_{\perp}<h_{\perp}^{c}$, and $\xi \sim \exp (+\Delta / T)]$, QC $(\xi \sim 1 / T)$, and QD $(g>1$, hence $h_{\perp}>h_{\perp}^{c}$, and $\xi \sim$ constant) [6]. The dashed and dotted lines represent the crossover temperature from the QC to the RC regime at $T \sim \Delta$ and from the QC to the QD regime at $T \sim|\Delta|$, respectively. An isolated 1D Ising chain would exhibit ferromagnetic long-range order only at $T=0$ below $h_{\perp}^{c}$, but the 3D interchain couplings lead to a 3D order at $T>0$ up to $h_{\perp}^{c, 3 D}$ $\left(>h_{\perp}^{c}\right.$ ). The filled circle at $T=0$ and the 1D critical field $h_{\perp}^{c}$ represents the QCP of the individual Ising chain.

A major advantage of working with the TFIC as a model system for testing the fundamental ideas of quantum phase transitions is that, in the absence of a transverse magnetic field $(g=0)$, the thermodynamic properties of the Ising chain can be rigorously solved at arbitrary temperatures [24]. Even in a finite transverse field $(g>0)$, the TFIC is well understood at $T=0[12,22,25]$, and quantum critical (QC) scaling theory extends the $T=0$ results to finite temperatures $[6,21]$.

We show the crystal structure of $\mathrm{CoNb}_{2} \mathrm{O}_{6}$ in Fig. 2 [26]. All the pictorial images of the crystal structure in this paper were drawn using VESTA [27]. The Co-O-Co chains propagate along the $\mathrm{c}$ axis, and the easy axis of the Co moments lies within the ac plane [14,15]. The ferromagnetic super-exchange interaction between the nearestneighbor Co ions is estimated to be $J=17-23 \mathrm{~K}$, based on ESR [18] and neutron scattering [20] measurements. From the disappearance of magnetic Bragg peaks in the transverse magnetic field applied along the $\mathrm{b}$ axis, the three-dimensional (3D) critical field was estimated to be $h_{\perp}^{c, 3 D}=5.5$ Tesla $[20,28]$. The interchain couplings between adjacent $\mathrm{Co}$ chains are antiferromagnetic $[14,20]$, weaker than $J$ by an order of magnitude $[18,20]$, and frustrated $[14,29]$. This means that the 3D magnetic long-range order induced by interchain interactions, which tends to mask the effects of the onedimensional (1D) QCP of the individual Ising chains, is suppressed; the 3D ordering temperature is as low as $T_{c}^{3 D}=2.9 \mathrm{~K}$ even in $h_{\perp}=0[14,15]$. Combined with the modest $J$, Ising chains in $\mathrm{CoNb}_{2} \mathrm{O}_{6}$ are ideal for testing the TFIC Hamiltonian, but they were overlooked for three decades.

In what follows, we will report on a ${ }^{93} \mathrm{Nb}$ nuclear magnetic resonance (NMR) investigation of quantum spin fluctuations in $\mathrm{CoNb}_{2} \mathrm{O}_{6}$. NMR is a powerful low-energy probe, and it is good at probing the physical properties near QCPs [30-38]. We will map the evolution of low-energy quantum fluctuations of Co spins near the QCP by taking advantage of the hyperfine interactions between Co electron spins and ${ }^{93} \mathrm{Nb}$ nuclear spins. We will experimentally verify the phase diagram of the TFIC in Fig. 1 above $T=0$ for the first time and demonstrate that the effect of the QCP persists at finite temperatures as high as $T \sim 0.4 \mathrm{~J}$.

\section{EXPERIMENTAL}

We grew the $\mathrm{CoNb}_{2} \mathrm{O}_{6}$ single crystal from a stoichiometric mixture of cobalt and niobium oxides using a floating zone furnace. We assessed the surface quality and oriented the crystal utilizing Laue x-ray diffractometry. Once the material was sectioned into oriented slices along the $\mathrm{a}, \mathrm{b}$, and $\mathrm{c}$ crystallographic directions, these were individually scanned with the Laue diffractometer, and they showed a uniform, single-crystalline structure. A small section of the single crystal was ground into a powder and analyzed using powder x-ray diffraction, which showed only single-phase cobalt niobate in the crystal within instrument resolution. The features present in the SQUID magnetometry data shown in Fig. 2(d) matched previously published data on this material [14].

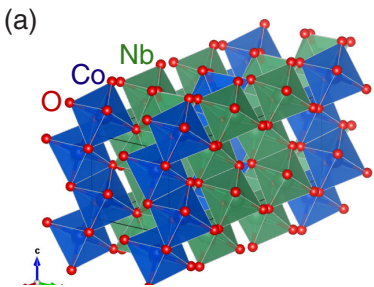

(b)

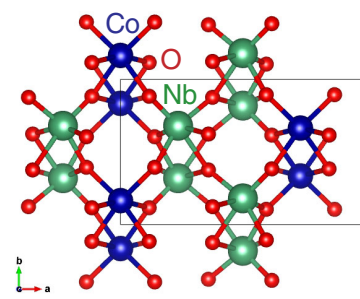

(c)
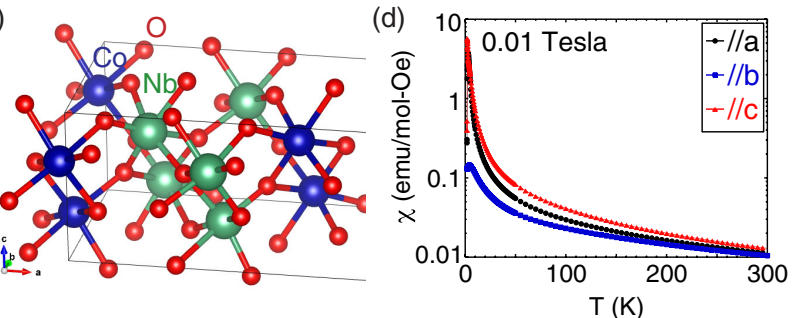

FIG. 2. (a) The crystal structure of $\mathrm{CoNb}_{2} \mathrm{O}_{6}$. (b) Both magnetic $\mathrm{CoO}_{6}$ and nonmagnetic $\mathrm{NbO}_{6}$ octahedra form a chain along the $\mathrm{c}$ axis, as seen from the $\mathrm{c}$-axis direction. The $\mathrm{Nb}-\mathrm{O}-\mathrm{Nb}$ chain is inside an isosceles triangle formed by three Co-O-Co chains. The transverse field $h_{\perp}$ is applied along the b axis. (c) Each $\mathrm{Nb}$ site is bonded with two Co-O-Co chains across $\mathrm{O}$ sites. (d) Bulk magnetic susceptibility $\chi$ data measured with SQUID in an external magnetic field of $0.01 \mathrm{~T}$. 
For NMR measurements, we cut a piece of single crystal with the approximate dimensions of $4 \mathrm{~mm} \times 2 \mathrm{~mm} \times 5 \mathrm{~mm}$. We glued the crystal to a sturdy sample holder made of machinable aluminum oxide (MACOR ceramic) with a thickness of around $3 \mathrm{~mm}$ to ensure that the crystal orientation did not change at low temperatures. We found that the strong magnetic torque applied to the crystal by the external magnetic field could easily bend sample holders made of soft materials such as plexiglass or plastic, and introduce noticeable systematic errors below around $10 \mathrm{~K}$.

We observed ${ }^{93} \mathrm{Nb}$ NMR in a broad range of temperature from $2 \mathrm{~K}$ (around $0.1 J$ ) up to $295 \mathrm{~K}$. We show the typical ${ }^{93} \mathrm{Nb}$ NMR spectrum in the inset of Fig. 3. Since the ${ }^{93} \mathrm{Nb}$ nuclear spin is $I=9 / 2$, we observed four pairs of satellite transitions split by a quadrupole frequency $\nu_{Q}^{b}=1.9 \mathrm{MHz}$, in addition to the large central peak arising from the $I_{z}=$ $+\frac{1}{2}$ to $-\frac{1}{2}$ transition. In the main panel of Fig. 3 , we also show the temperature dependence of the central transition in $h_{\perp}=5.3$ Tesla applied along the $\mathrm{b}$ axis.

We measured the ${ }^{93} \mathrm{Nb}$ longitudinal relaxation rate $1 / T_{1}$ by applying an inversion $\pi$ pulse prior to the $\pi / 2-\pi$ spinecho sequence and by monitoring the recovery of the spinecho intensity $M(t)$ as a function of the delay time $t$. The typical width of the $\pi / 2$ pulse was around $1 \mu \mathrm{s}$. We fit these recovery curves to the solutions of the rate equation [39]:

$$
M(t)=M(\infty)-A \sum_{j=1}^{9} a_{j} e^{-b_{j} t / T_{1}},
$$

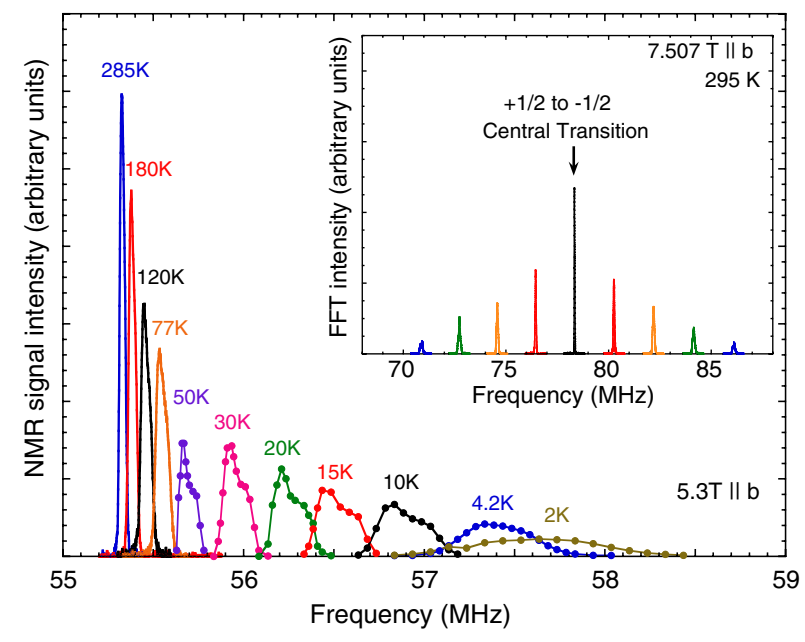

FIG. 3. The temperature dependence of the ${ }^{93} \mathrm{Nb}$ NMR line shape observed for the central transition between the $I_{z}=+\frac{1}{2}$ and $-\frac{1}{2}$ energy levels in $h_{\perp}=5.3$ Tesla applied along the $\mathrm{b}$ axis. We obtained the line shapes using the FFT of the spinecho signal above $77 \mathrm{~K}$. For the broader line shapes below $77 \mathrm{~K}$, we measured the integral of the spin echo as a function of the frequency. Inset: The ${ }^{93} \mathrm{Nb}$ NMR line shape at $295 \mathrm{~K}$ observed at $7.507 \mathrm{~T}$ using the FFT of spin-echo signals. The largest peak in the middle is the central transition, and four additional pairs of weaker peaks arise from $I_{z}=m$ to $m+1$ transitions $(m=-9 / 2,-7 / 2,-5 / 2,-3 / 2,+1 / 2, \quad+3 / 2$, $+5 / 2$, and $+7 / 2)$. with three free parameters: $M(\infty), A$, and $1 / T_{1}$. By solving the coupled rate equations for $I=\frac{9}{2}$ under the appropriate initial condition, one can calculate and fix the coefficients as $\left(a_{1}, a_{2}, a_{3}, a_{4}, a_{5}, a_{6}, a_{7}, a_{8}, a_{9}\right)=(0.653,0,0.215,0$, $0.092,0,0.034,0,0.06)$ for the central transition and $(0.001,0.0112,0.0538,0.1485,0.2564,0.2797,0.1828$, $0.0606,0.0061$ ) for the $I_{z}= \pm \frac{7}{2}$ to $I_{z}= \pm \frac{9}{2}$ fourth satellite transitions, while $\left(b_{1}, b_{2}, b_{3}, b_{4}, b_{5}, b_{6}, b_{7}, b_{8}, b_{9}\right)=$ $(45,36,28,21,15,10,6,3,1)$ for both cases [39].

An example of the signal recovery of the central transition observed at $130 \mathrm{~K}$ in $h_{\perp}=3$ Tesla is shown in Fig. 4, in comparison to that observed for a fourth satellite transition on the higher-frequency side. Our results in Fig. 4 confirm that the best-fit values of $1 / T_{1}$ agree within around $2 \%$ between the central and satellite transitions. The central transition is the strongest among all nine peaks, as shown in the inset of Fig. 3, and hence most advantageous in terms of the signal intensity. When the relaxation rate exceeds $1 / T_{1} \sim 2 \times 10^{3} \mathrm{~s}^{-1}$, however, accurate measurements of $1 / T_{1}$ using the central transition become increasingly difficult because the recovery curve $M(t)$ is dominated by two extremely fast normal modes, $0.653 e^{-45 t / T_{1}}+0.215 e^{-28 t / T_{1}}$; the signal intensity $M(t)$ begins to recover at a time scale comparable to the inversion pulse width. Accordingly, measurements of $1 / T_{1}$ using the fourth satellite transition become more advantageous in the low-temperature, low-field regime because the recovery curve is dominated by slower normal modes, $0.256 e^{-15 t / T_{1}}+0.279 e^{-10 t / T_{1}}$. We present an

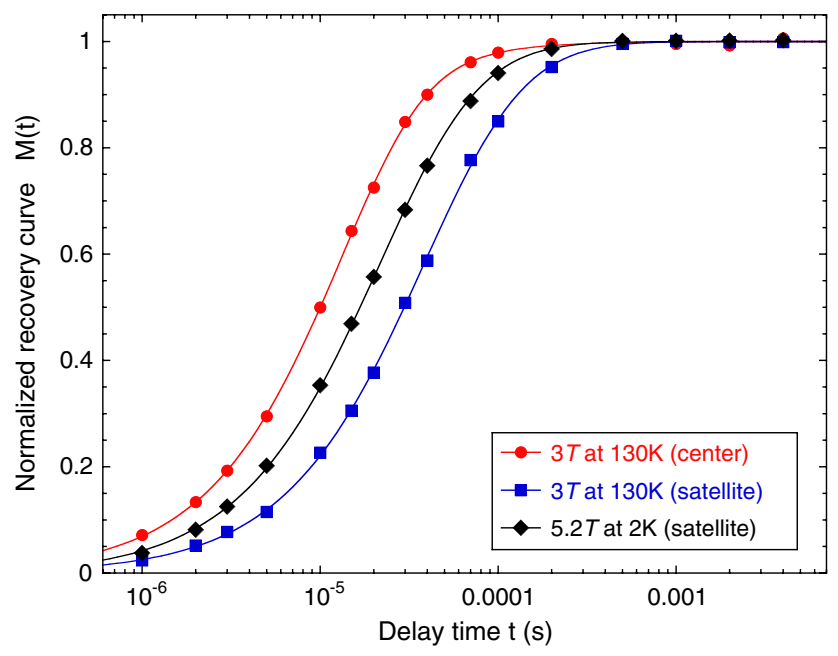

FIG. 4. Examples of the recovery of the spin-echo intensity, $M(t)$, observed for the central and fourth satellite transitions at $130 \mathrm{~K}$ in $h_{\perp}=3$ Tesla. For comparison, we normalized the recovery curves by plotting $1-[M(\infty)-M(t)] / A$ as a function of $t$. The solid lines represent the best fit with $1 / T_{1}=1.99 \times$ $10^{3} \mathrm{~s}^{-1}$ for the central transition and $1 / T_{1}=1.96 \times 10^{3} \mathrm{~s}^{-1}$ for the fourth satellite transition, as described in the text. Also plotted is the recovery curve observed for the fourth satellite peak at $2 \mathrm{~K}$ in $h_{\perp}=5.2$ Tesla. 
additional example of the $1 / T_{1}$ measurement using the fourth satellite at $2 \mathrm{~K}$ and $h_{\perp}=5.2$ Tesla in Fig. 4 .

\section{RESULTS AND DISCUSSIONS}

\section{A. $T$ and $h_{\perp}$ dependences of $1 / T_{1}$}

In Fig. 5, we summarize the $T$ and $h_{\perp}$ dependences of $1 / T_{1}$. Notice that $1 / T_{1}$ varies by more than 3 orders of magnitude between $h_{\perp}=3$ and $9 \mathrm{~T}$. Quite generally, $1 / T_{1}$ probes the wave-vector $\mathbf{k}$ integral within the first Brillouin zone of the dynamical spin structure factor $S\left(\mathbf{k}, \omega_{n}\right)$ at the NMR frequency $\omega_{n} / 2 \pi$ (around $50 \mathrm{MHz}$ ):

$$
1 / T_{1}=\sum_{\mathbf{k}}\left|a_{\mathrm{hf}}\right|^{2} S\left(\mathbf{k}, \omega_{n}\right),
$$

where $a_{\mathrm{hf}}$ is the hyperfine coupling between the observed nuclear spin and Pauli matrices. In essence, $1 / T_{1}$ measures the strength of Co spin fluctuations at the time scale set by the NMR frequency.

Our $1 / T_{1}$ data in Fig. 5 exhibit two distinct field regimes at low temperatures because the spin excitation spectrum changes its character across $h_{\perp}^{c}$, as summarized in Fig. 6. Below $h_{\perp}^{c} \sim 5.3$ Tesla, $1 / T_{1}$ diverges gradually toward $T=0$, signaling the critical slowing down of Co spin fluctuations in the renormalized classical (RC) [2] regime of Fig. 1 toward the $T=0$ ferromagnetic ground state of each individual Ising chain. In other words, the spectral weight of the Co spin-spin correlation function grows at the quasielastic peak located at $k=0$ in Fig. 6(a), below $h_{\perp}^{c} \sim 5.3$ Tesla. The Co spin-spin correlation length $\xi$ along the chain grows as $\xi \sim \exp (+\Delta / T)$ in the $\mathrm{RC}$ regime [6], where $\Delta$ is the gap in the spin excitation

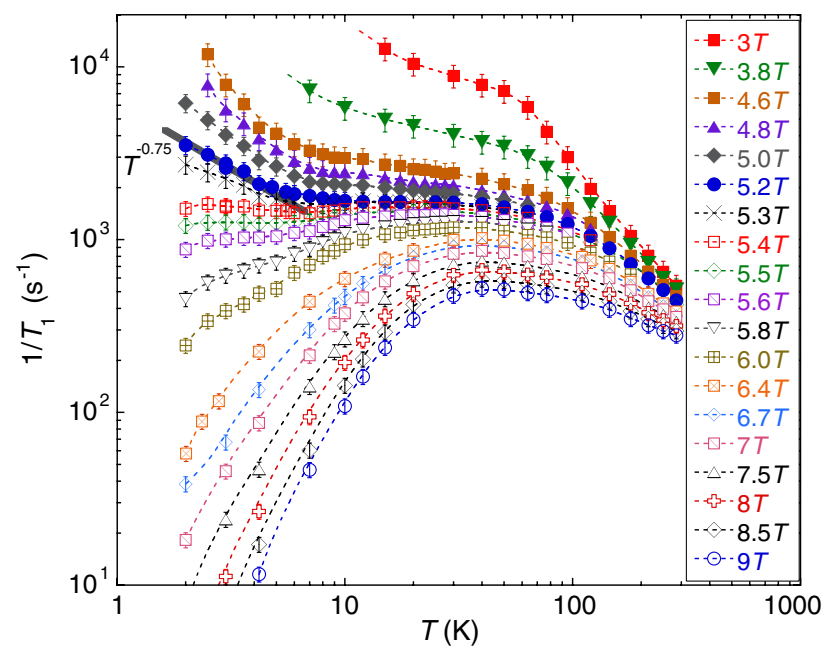

FIG. 5. The temperature dependence of $1 / T_{1}$ in $h_{\perp}$ applied along the $\mathrm{b}$ axis. All dashed lines interconnecting the data points are guides for the eye. The black solid line through the data points measured at 5.2 Tesla represents a power-law fit, $1 / T_{1} \sim 6.2 \times 10^{3} T^{-0.75} \mathrm{~s}^{-1}$.
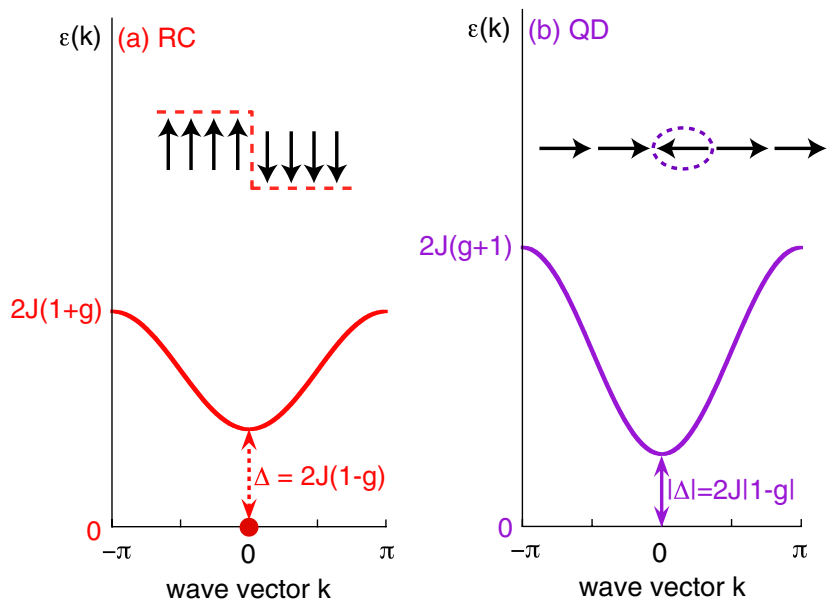

FIG. 6. (a) The two components of the spin excitation spectrum in the $\mathrm{RC}$ regime: the quasielastic peak at the origin (represented by a filled dot) and the propagating domain walls, as schematically shown in the inset. The dispersion of the latter (solid curve) is $\epsilon(k)=J\left[2-2 g \cos (k)+O\left(g^{2}\right)\right]$, with an excitation gap $\Delta=$ $2 J(1-g)[6]$. The quasielastic peak becomes a Bragg peak when $\xi$ diverges toward the 1D ferromagnetic long-range order at $T=0$. Since NMR is a low-energy probe, our $1 / T_{1}$ data measured below $h_{\perp}^{c}$ probe the quasielastic mode. (b) The spin excitation spectrum in the QD regime, $\epsilon(k)=J g[2-$ $\left.(2 / g) \cos (k)+O\left(1 / g^{2}\right)\right]$ with a gap $|\Delta|=2|1-g|[6]$, which arises from the propagation of flipped spins (inset). Unlike the RC regime, there is no quasielastic peak.

spectrum, as defined in Fig. 6(a). Accordingly, we expect $1 / T_{1} \sim \exp (+\Delta / T)$ for $T \ll \Delta$. We summarize the details of the theoretical expressions of $1 / T_{1}$ for the TFIC in Appendix A.

In contrast, $1 / T_{1}$ observed above $h_{\perp}^{c} \sim 5.3$ Tesla saturates and begins to decrease with temperature. We recall that the $T=0$ ground state remains paramagnetic in the quantum disordered (QD) regime above $h_{\perp}^{c}$, as shown in Fig. 1, and hence there is no quasielastic mode of spin excitations in Fig. 6(b). The latter implies that $1 / T_{1}$ in the QD regime is dominated by the thermal activation of spin excitations across the gap, $|\Delta|$. Therefore, we expect $1 / T_{1} \sim \exp (-|\Delta| / T)$ for $T \ll|\Delta|$. We have thus identified the 1D QCP (one-dimensional QC point) of each individual Ising chain as $h_{\perp}^{c} \sim 5.3$ Tesla.

\section{B. Estimation of the spin excitation gap $\Delta$}

In Fig. 7(a), we present the exponential fit of $1 / T_{1} \sim \exp (\Delta / T)$, with $\Delta$ as a free parameter. We summarize the $h_{\perp}$ dependence of $\Delta$ in Fig. 7(b). The fitting range barely satisfies $T<|\Delta|$ near $h_{\perp} \sim 5.3$ Tesla, limiting the accuracy of our estimation of $\Delta$. To improve the accuracy, we constructed the scaling plots of $T^{+0.75} / T_{1}$ as a function of $\Delta / T$ in Fig. 8. We first estimated the magnitude of $\Delta$ from Fig. 7(a). Subsequently, for the field range between 5.0 and $6.7 \mathrm{~T}$, we made slight adjustments to 

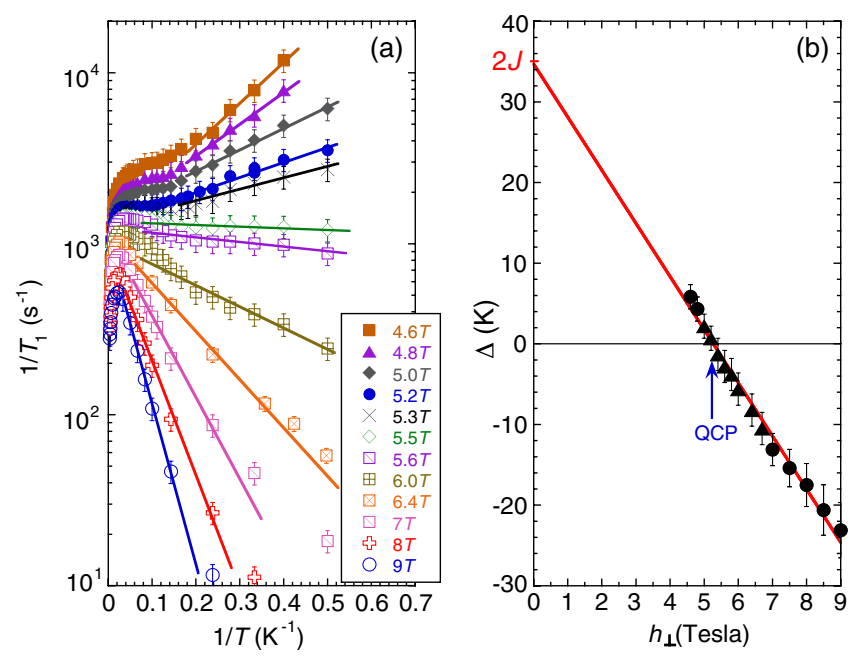

FIG. 7. Estimation of the gap $\Delta$. (a) The exponential fit $1 / T_{1} \sim$ $\exp (\Delta / T)$ for representative values of $h_{\perp}$. (b) Filled circle represents $\Delta$ as determined from (a), while filled triangle is based on the scaling analysis. Also shown is a linear fit, $\Delta=2 J\left(1-h_{\perp} / h_{\perp}^{c}\right)$. From the fit, we estimate $h_{\perp}^{c}=5.25 \pm$ 0.15 Tesla and $J=17.5_{-1.5}^{+2.5} \mathrm{~K}$.

the magnitude of $\Delta$ to improve the scaling collapse in Fig. 8. The final results of $\Delta$ thus estimated from Fig. 8 are presented in Fig. 7(b) using filled triangle. We note that this procedure changes the estimated value of $\Delta$ only by a few K.

Remarkably, we found that $\Delta$ varies linearly with $h_{\perp}$. This linear behavior is precisely what we expect from the theoretical prediction for the nearest-neighbor quantum Ising chain, $\Delta=2 J\left(1-h_{\perp} / h_{\perp}^{c}\right)[6]$. From the intercept of
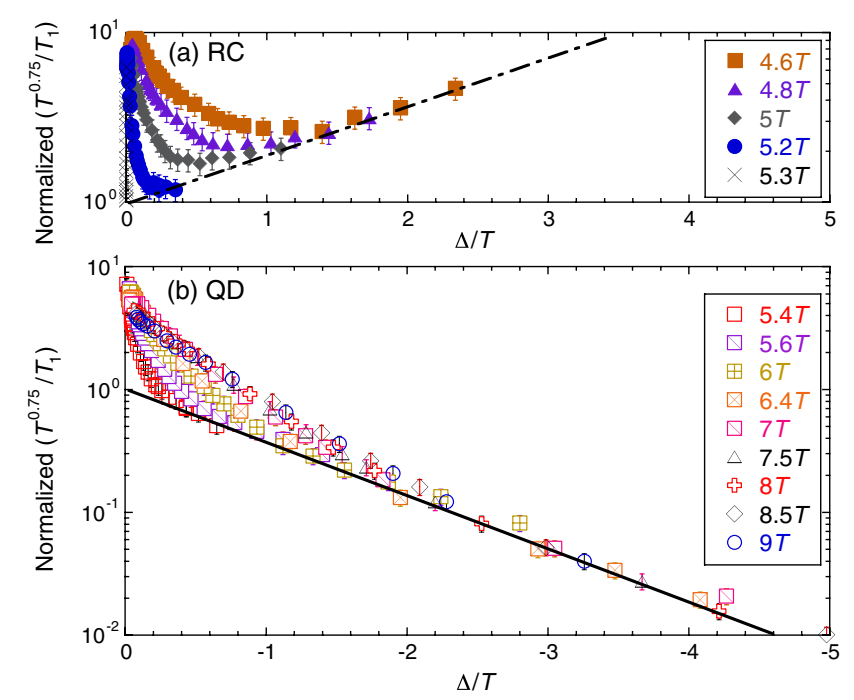

FIG. 8. The scaling plots of $T^{+0.75} / T_{1}$ as a function of $\Delta / T$ in (a) the RC regime and (b) the QD regime. For clarity, we normalized the overall magnitude of $T^{+0.75} / T_{1}$ as unity for the QC regime. The dashed-dotted line in (a) is a guide for the eyes, while the solid line in (b) represents $1 / T_{1} \propto e^{-|\Delta| / T}$. the linear fit with the horizontal axes, we estimate $h_{\perp}^{c}=5.25 \pm 0.15$ Tesla. This $1 \mathrm{D}$ critical field observed by our NMR measurements agrees very well with the earlier observation of the saturation of the so-called $E_{8}$ golden ratio [20]. From the intercept of the linear fit with the vertical axis, we also estimate $J=17.5_{-1.5}^{+2.5} \mathrm{~K}$, in excellent agreement with earlier reports based on ESR [18] and neutron scattering [20].

\section{Phase diagram of the TFIC in $\mathrm{CoNb}_{2} \mathrm{O}_{6}$}

We present the color plot of $1 / T_{1}$ in Fig. 9. Also shown in Fig. 9 are the crossover temperatures, $\Delta$ and $|\Delta|$, based on the linear fit in Fig. 7(b). Our color plot visually captures the crossover from the QC regime to the RC and QD regimes. We are the first to verify the theoretical $T-h_{\perp}$ phase diagram in Fig. 1 for finite temperatures, $T>0$, using an actual material.

\section{Quantum criticality of the TFIC at finite temperatures}

Having established the phase diagram of the TFIC in $\mathrm{CoNb}_{2} \mathrm{O}_{6}$, we are ready to test the finite-temperature properties of the QC regime located between the RC and QD regimes. At the 1D critical field $h_{\perp}^{c}$, we applied QC scaling to Eq. (3) and obtained

$$
1 / T_{1}=2.13\left|a_{\mathrm{hf}}\right|^{2} J^{-0.25} T^{-0.75},
$$

for the nearest-neighbor TFIC [see Eq. (A7) for the details]. We determined the hyperfine form factor $\left|a_{\mathrm{hf}}\right|^{2}$ based on the ${ }^{93} \mathrm{Nb} \mathrm{NMR}$ frequency-shift measurements and used Eq. (4) to estimate $1 / T_{1}=(4.2-8.4) \times 10^{3} T^{-0.75} \mathrm{~s}^{-1}$ at finite temperatures above the QCP without any adjustable

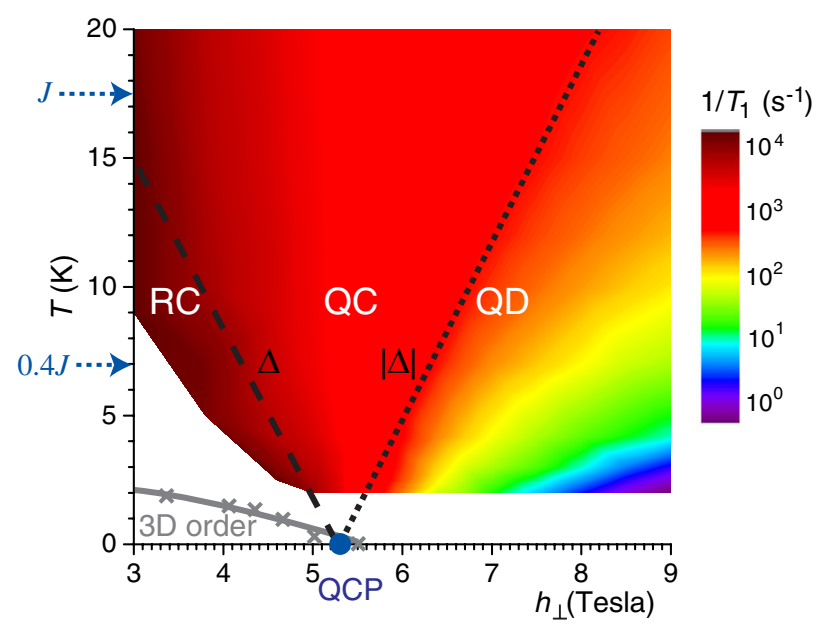

FIG. 9. A color plot of $1 / T_{1}$. The dashed (dotted) line represents the expected crossover temperature $\Delta(|\Delta|)$ from the QC to the RC (QD) regime, based on the linear $h_{\perp}$ dependence of $\Delta$ estimated in Fig. 7(b). Also shown (grey x) is the 3D ordering temperature $T_{c}^{3 D}[28]$. 
parameters. We refer readers to Appendix B for the details of the data analysis. This parameter-free prediction is in excellent quantitative agreement with our experimental finding, $1 / T_{1} \sim 6.2 \times 10^{3} T^{-0.75} \mathrm{~s}^{-1}$, as shown by a solid line in Fig. 5 through the data points observed at $5.2 \mathrm{~T}$. Thus the QC scaling theory accounts for the low-frequency spin dynamics of the TFIC above $T=0$ at a quantitative level.

It is equally important to realize that $1 / T_{1}$ data exhibit the expected power-law behavior, $1 / T_{1} \sim T^{-0.75}$, up to $\sim 7 \mathrm{~K}$, which corresponds to $T \sim 0.4 \mathrm{~J}$. Our finding therefore addresses an important and unresolved question that the strongly correlated electrons community has faced for years: How high in temperature does the effect of the QCP persist? For the TFIC, the quantum fluctuations originating from the zero-temperature QCP persist up to as high as $T \sim 0.4 J$. Our experimental finding is consistent with the earlier theoretical report that the QC scaling holds up to $T \sim 0.5 J$ for the TFIC [11].

\section{SUMMARY AND CONCLUSIONS}

Using the quasi one-dimensional Co chains in $\mathrm{CoNb}_{2} \mathrm{O}_{6}$, we experimentally tested the quantum criticality of the TFIC at finite temperatures above $T=0$ for the first time. Based on the measurements of the ${ }^{93} \mathrm{Nb}$ longitudinal relaxation rate $1 / T_{1}$, we identified the distinct behaviors of low-frequency spin fluctuations in the RC, QC, and QD scaling regimes of the TFIC and constructed the $T-h_{\perp}$ phase diagram of the TFIC in Fig. 9. We observed no evidence for a crossover into the $3 \mathrm{D}$ regime in the temperature and field range of our concern. We also reported the transverse field $\left(h_{\perp}\right)$ dependence of the spin excitation gap parameter $\Delta$ in Fig. 7(b); our results exhibit a linear dependence on $h_{\perp}$, in agreement with the theoretical prediction for the nearest-neighbor TFIC. Our $1 / T_{1}$ data observed for the QC regime near $h_{\perp}^{c} \approx 5.25 \mathrm{~T}$ exhibit the expected mild power-law divergence, 1/ $T_{1} \sim T^{-0.75}$, toward the quantum critical point at $T=0$. Furthermore, the parameter-free prediction based on quantum critical scaling reproduces the magnitude of $1 / T_{1}$ within about $\pm 36 \%$. Our results in Fig. 5 establish that the quantum critical behavior persists to as high as $T \sim 0.4 J$. To the best of our knowledge, this is the first example of the quantitative test of the finite-temperature effects on quantum criticality for model Hamiltonians with a rigorously solvable ground state.

We mark the upper bound of the QC scaling regime, $T \sim 0.4 J$, in Fig. 9 with a horizontal arrow. Such a robust quantum criticality observed at finite temperatures above the QCP is in stark contrast with the case of thermally induced classical phase transitions; the critical region of the latter generally narrows as the phase-transition temperature approaches zero, and eventually diminishes at $T=0$ [10]. Many authors have constructed analogous color plots for different parameters (such as electrical resistivity) for a variety of strongly correlated electron systems, including copper-oxide and iron-pnictide high- $T_{c}$ superconductors and heavy-fermion systems $[8,9]$. The aim of these authors was to build a circumstantial case that quantum fluctuations persist at finite temperatures far above the QCP. The overall similarity between our Fig. 9 and the case of high- $T_{c}$ cuprates and other exotic superconductors gives us hope that quantum fluctuations may indeed account for the mechanism of exotic superconductivity.

\section{ACKNOWLEDGMENTS}

T. I. and S. S. thank A. P. Young, Y. Itoh, B. D. Gaulin, M. P. Gelfand, S.-S. Lee, T. Sakai, and H. Nojiri for helpful communications. The work at McMaster was supported by NSERC and CIFAR. S. S. acknowledges financial support from NSF DMR-1103860.

Note added.-After the initial submission of this work, a theoretical prediction was made for the temperature dependence of $1 / T_{1}$ under the presence of an internal longitudinal magnetic field in the three-dimensionally ordered state [40]. The three-dimensional effects [29,40], however, are beyond the scope of the present work.

\section{APPENDIX A: THEORETICAL DERIVATION OF $1 / T_{1}$ IN THE QUANTUM ISING CHAIN}

Here, we summarize the derivations of the theoretical expressions of $1 / T_{1}$ in the TFIC. Our notation will be the same as in Ref. [6]. Some results will be specific to the nearest-neighbor Ising model, but most are more generally applicable to the vicinity of the quantum critical point of a generic one-dimensional Ising chain. In general, the NMR relaxation rate is defined by

$$
\begin{aligned}
\frac{1}{T_{1}} & =\lim _{\omega \rightarrow 0} \frac{2 T}{\omega} \int \frac{d k}{2 \pi}\left|a_{\mathrm{hf}}\right|^{2} \operatorname{Im} \chi(k, \omega), \\
& =\int \frac{d k}{2 \pi}\left|a_{\mathrm{hf}}\right|^{2} \mathrm{~S}(k, \omega=0), \\
& =\int_{-\infty}^{+\infty} d t\left|a_{\mathrm{hf}}\right|^{2} \mathrm{C}(x=0, t),
\end{aligned}
$$

where $a_{\mathrm{hf}}$ represents the hyperfine coupling between the nuclear spin and the Pauli matrices $\sigma$, as defined by the hyperfine Hamiltonian $\hat{H}_{\mathrm{hf}}=\hat{I} \cdot a_{\mathrm{hf}} \cdot \hat{\sigma}$. We define the correlation function for Pauli matrices, and $\hbar=k_{B}=1$ unless noted otherwise.

\section{Renormalized classical regime}

This region is characterized by an energy gap $\Delta \sim\left(g_{c}-g\right)$ and a $T=0$ ordered moment $N_{o} \sim\left(g_{c}-g\right)^{1 / 8}$. $N_{o}$ represents the ordered moment of an Ising chain at $T=0$ and should not be confused with the $3 \mathrm{D}$ ordered moment 
induced by interchain couplings. By expressing our results in terms of $\Delta$ and $N_{o}$, they are generally valid beyond the nearest-neighbor model. For the specific case of the nearest-neighbor model, we have $\Delta=2 J(1-g)$ and $N_{o}=\left(1-g^{2}\right)^{1 / 8}$. The result for $C(x, t)$ may be found below (4.81) in Ref. [6], and this leads to

$$
\frac{1}{T_{1}}=\left|a_{\mathrm{hf}}\right|^{2} \frac{\pi N_{o}^{2}}{T} e^{+\Delta / T} .
$$

Notice that $1 / T_{1}$ is expected to diverge exponentially, even though there is an energy gap $\Delta$ in the excitation spectrum of the domain-wall quasiparticles. This is because NMR is a low-energy probe, and $1 / T_{1}$ in the $\mathrm{RC}$ regime is dominated by the low-frequency spin fluctuations associated with the quasielastic mode of the $1 \mathrm{D}$ Ising chain induced by ferromagnetic short-range order.

Our scaling analysis in Fig. 8(a) suggests that the observed divergent behavior of $1 / T_{1}$ is somewhat weaker than $\frac{1}{T_{1}} \sim \frac{1}{T} e^{+\Delta / T}$, perhaps because our experimental range of $T$ and $h_{\perp}$ is not deep inside the RC regime, or possibly because of the influence of additional terms in the Hamiltonian neglected in the theoretical calculations. Accordingly, we fit the $1 / T_{1}$ data in the RC regime with the simple exponential form, $1 / T_{1} \propto e^{+\Delta / T}$, in Fig. 7(a), ignoring the temperature-dependent prefactor $\sim 1 / T$.

\section{Quantum critical regime}

Here, we have, in imaginary time $\tau$, from (4.106) in Ref. [6], that

$$
C(x=0, \tau)=Z T^{1 / 4} \frac{G_{I}(0)}{[2 \sin (\pi T \tau)]^{1 / 4}},
$$

where $G_{I}(0)=0.858714569$ and

$$
Z=\lim _{\Delta \rightarrow 0} \frac{N_{o}^{2}}{\Delta^{1 / 4}}
$$

the value of $Z$ is a general result when approaching from the ordered side, valid beyond the nearest-neighbor model.

From Eq. (A3), we have the local susceptibility in imaginary time,

$$
\chi\left(x=0, \omega_{n}\right)=\int_{0}^{1 / T} d \tau e^{i \omega_{n} \tau} C(x=0, \tau) .
$$

We evaluate the Fourier transform using Eqs. (3.12), (3.22), and (3.24) of Ref. [41], and we obtain

$$
\begin{aligned}
\operatorname{Im} \chi\left(x=0, \omega_{n}\right)= & \frac{Z G_{I}(0)}{T^{3 / 4} 2^{1 / 4} \sqrt{\pi} \Gamma(1 / 8) \Gamma(5 / 8)} \\
& \times \sinh \left(\frac{\omega}{2 T}\right)\left|\Gamma\left(\frac{1}{8}-\frac{i \omega}{2 \pi T}\right)\right|^{2} .
\end{aligned}
$$

This gives us

$$
\frac{1}{T_{1}}=\left|a_{\mathrm{hf}}\right|^{2} \frac{Z}{T^{3 / 4}} \frac{G_{I}(0) \Gamma(1 / 8)}{2^{1 / 4} \sqrt{\pi} \Gamma(5 / 8)}=2.13\left|a_{\mathrm{hf}}\right|^{2} \frac{Z}{T^{3 / 4}} .
$$

In the case of the nearest-neighbor Ising model, $\Delta=$ $2 J(1-g)$ and $N_{o}=\left(1-g^{2}\right)^{1 / 8}$. Accordingly, we obtain $Z=J^{-1 / 4}$ from Eq. (A4), and hence Eq. (A7) leads to Eq. (4) in the main text.

\section{Quantum disordered regime}

Here, we expect that $1 / T_{1}$ diminishes exponentially in the quantum disordered regime because of the excitation gap $|\Delta|$, and so

$$
\frac{1}{T_{1}} \propto e^{-|\Delta| / T}
$$

where now $\Delta<0$. However, there is no explicit computation in the TFIC establishing this, and the prefactor is unknown. Accordingly, we fit the $1 / T_{1}$ data in Fig. 7 (a) to the simple activation form.

\section{APPENDIX B: ANALYSIS OF $1 / T_{1}$ IN THE QC REGIME}

In the previous section, we defined the hyperfine coupling with Pauli matrices as $a_{\mathrm{hf}}$ to maintain consistency of the notation for the dynamical spin susceptibility defined in Ref. [6]. To use the standard notations of NMR data analysis, here we introduce the hyperfine coupling $A_{\mathrm{hf}}$ between the nuclear spin $I$ and electron spin $S$ through the hyperfine Hamiltonian $\hat{H}_{\mathrm{hf}}=\hat{I} \cdot A_{\mathrm{hf}} \cdot \hat{S}$. That is, $a_{\mathrm{hf}}=S A_{\mathrm{hf}}$. Earlier ESR measurements determined the anisotropic $g$ tensor of the $\mathrm{Co}^{2+}$ ions in $\mathrm{CoNb}_{2} \mathrm{O}_{6}$ as $g^{(a)}=4.3$ and $g^{(c)}=6.1$ by taking the Co pseudospin as $S=\frac{1}{2}[18]$.

Recalling that $1 / T_{1}$ measured with an external magnetic field applied along the crystal $\mathrm{b}$ axis probes the fluctuating hyperfine fields along the a and c axes, we may rewrite Eq. (A7) as

$$
\frac{1}{T_{1}}=2.13 S^{2} \frac{\left|A_{\mathrm{hf}}^{(a)} / \hbar\right|^{2}+\left|A_{\mathrm{hf}}^{(c)} / \hbar\right|^{2}}{2} \frac{\hbar}{\left(k_{B} J\right)^{1 / 4}\left(k_{B} T\right)^{3 / 4}},
$$

where we show $\hbar$ and $k_{B}$ explicitly.

Next, we estimate the uniform $\mathbf{k}=\mathbf{0}$ component of the hyperfine coupling from the NMR frequency shift $K$ [42],

$$
K^{(\alpha)}=\frac{A_{\mathrm{hf}}^{(\alpha)}(\mathbf{k}=\mathbf{0})}{g^{(\alpha)} \mu_{B}} \chi^{(\alpha)}+K_{\mathrm{chem}}^{(\alpha)}
$$

where $\alpha=a, \mathrm{~b}$, and $\mathrm{c}$, and $K_{\mathrm{chem}}^{(\alpha)}$ is the small temperatureindependent chemical shift. Accordingly, 


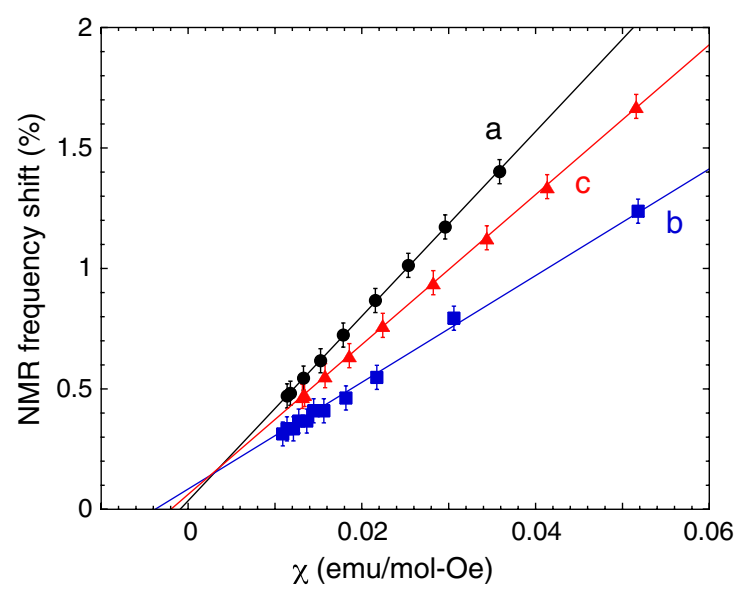

FIG. 10. The NMR frequency shift $K^{(\alpha)}$ vs the bulk magnetic susceptibility $\chi^{(\alpha)}$, with $T$ as the implicit parameter $(\alpha=a$, b, or c). The straight lines are the best linear fits.

$$
\frac{A_{\mathrm{hf}}^{(\alpha)}(\mathbf{k}=\mathbf{0})}{\hbar}=\gamma_{n} N_{A} g^{(\alpha)} \mu_{B} \frac{d K^{(\alpha)}}{d \chi^{(\alpha)}}
$$

where the ${ }^{93} \mathrm{Nb}$ nuclear gyromagnetic ratio is $\gamma_{n} / 2 \pi=10.407 \mathrm{MHz} /$ Tesla, and $N_{A}$ is Avogadro's number.

To determine the only unknown parameter $\frac{d K^{(\alpha)}}{d \chi^{(\alpha)}}$ in the right-hand side of Eq. (B3), we plot $K^{(\alpha)}$ in Fig. 10 as a function of the molar magnetic susceptibility $\chi^{(\alpha)}$ measured along the corresponding orientations [see Fig. 2(d)], choosing $T$ as the implicit parameter. From the linear fit of the $K$ vs $\chi$ plot, we estimate the slope as $\frac{d K^{(\alpha)}}{d \chi^{(\alpha)}}=0.386$, 0.221 , and 0.311 for $\alpha=a, \mathrm{~b}$, and c, respectively. Therefore, we arrive at $A_{\mathrm{hf}}^{(a)}(\mathbf{k}=\mathbf{0}) / \hbar=6.0 \times 10^{7}\left(\mathrm{~s}^{-1}\right)$ and $A_{\mathrm{hf}}^{(c)}(\mathbf{k}=\mathbf{0}) / \hbar=7.0 \times 10^{7}\left(\mathrm{~s}^{-1}\right)$.

Next, we need to relate these results with the fluctuating hyperfine fields $\left|A_{\mathrm{hf}}^{(\alpha)} / \hbar\right|^{2}$ in Eq. (B1). The upper bound of the latter may be easily estimated as

$$
\left|A_{\mathrm{hf}}^{(\alpha)} / \hbar\right|^{2}=\left|A_{\mathrm{hf}}^{(\alpha)}(\mathbf{k}=\mathbf{0}) / \hbar\right|^{2},
$$

where we assumed that all Co chains fluctuate coherently with ferromagnetic interchain correlations. Inserting Eq. (B4) into Eq. (B1), we obtain $1 / T_{1}=8.4 \times$ $10^{3} T^{-0.75}\left(\mathrm{~s}^{-1}\right)$. This theoretical upper bound overestimates the experimental results observed for about $5.2 \mathrm{~T}$ by around $36 \%$.

In reality, the interchain couplings are smaller than $J$ by an order of magnitude, and they are frustrated. Since we are concerned with the temperature range $T>0.1 J$, it is safe to assume that the fluctuating transferred hyperfine fields from two nearby Co-O-Co chains are uncorrelated. Assuming that the magnitudes of these couplings are comparable $\left[\sim A_{\mathrm{hf}}^{(\alpha)}(\mathbf{k}=\mathbf{0}) / 2 \hbar\right]$ and that their fluctuations are additive, we arrive at

$$
\left|A_{\mathrm{hf}}^{(\alpha)} / \hbar\right|^{2} \sim 2 \times\left|A_{\mathrm{hf}}^{(\alpha)}(\mathbf{k}=\mathbf{0}) / 2 \hbar\right|^{2} .
$$

By inserting Eq. (B5) into Eq. (B1), we estimate $\frac{1}{T_{1}}=4.2 \times 10^{3} T^{-0.75}\left(\mathrm{~s}^{-1}\right)$. This underestimates the experimental observation by about $33 \%$.

[1] J. A. Hertz, Quantum Critical Phenomena, Phys. Rev. B 14, 1165 (1976).

[2] S. Chakravarty, B. I. Halperin, and D. R. Nelson, TwoDimensional Quantum Heisenberg Antiferromagnet at Low Temperatures, Phys. Rev. B 39, 2344 (1989).

[3] A. J. Millis, Effect of a Nonzero Temperature on Quantum Critical Points in Itinerant Fermion Systems, Phys. Rev. B 48, 7183 (1993).

[4] A. V. Chubukov, S. Sachdev, and J. Ye, Theory of Two-Dimensional Quantum Heisenberg Antiferromagnets with a Nearly Critical Ground State, Phys. Rev. B 49, 11919 (1994).

[5] A. M. Goldman and N. Markovic, SuperconductorInsulator Transitions in the Two-Dimensional Limit, Phys. Today 51, 39 (1998).

[6] S. Sachdev, Quantum Phase Transitions (Cambridge University Press, Cambridge, England, 1999), 1st ed.

[7] S. Sachdev, Quantum Magnetism and Criticality, Nat. Phys. 4, 173 (2008).

[8] P. Gegenwart, Q. Si, and F. Steglich, Quantum Criticality in Heavy-Fermion Metals, Nat. Phys. 4, 186 (2008).

[9] S. Sachdev and B. Keimer, Quantum Criticality, Phys. Today 64, 29 (2011).

[10] G. R. Lonzarich, Magnetic Quantum Liquid Enigma, Nat. Phys. 1, 11 (2005).

[11] A. Kopp and S. Chakravarty, Criticality in Correlated Quantum Matter, Nat. Phys. 1, 53 (2005).

[12] P. Pfeuty, The One-Dimensional Ising Model with a Transverse Field, Ann. Phys. (N.Y.) 57, 79 (1970).

[13] I. Maartense, I. Yaeger, and B. M. Wanklyn, Field-Induced Magnetic Transitions of $\mathrm{CoNb}_{2} \mathrm{O}_{6}$ in the Ordered State, Solid State Commun. 21, 93 (1977).

[14] W. Scharf, H. Weitzel, I. Yaeger, I. Maartense, and B. M. Wanklyn, Magnetic Structures of $\mathrm{CoNb}_{2} \mathrm{O}_{6}$, J. Magn. Magn. Mater. 13, 121 (1979).

[15] T. Hanawa, K. Shinkawa, M. Ishikawa, K. Miyatani, K. Saito, and K. Kohn, Anisotropic Specific Heat of $\mathrm{CoNb}_{2} \mathrm{O}_{6}$ in Magnetic Fields, J. Phys. Soc. Jpn. 63, 2706 (1994).

[16] C. Heid, H. Weitzel, P. Burlet, M. Bonnet, W. Gonschorek, T. Vogt, J. Norwig, and H. Fuess, Magnetic Phase Diagram of $\mathrm{CoNb}_{2} \mathrm{O}_{6}$ : A Neutron Diffraction Study, J. Magn. Magn. Mater. 151, 123 (1995).

[17] S. Kobayashi, S. Mitsuda, M. Ishikawa, K. Miyatani, and K. Kohn, Three-Dimensional Magnetic Ordering in the QuasiOne-Dimensional Ising Magnet $\mathrm{CoNb}_{2} \mathrm{O}_{6}$ with Partially Released Geometrical Frustration, Phys. Rev. B 60, 3331 (1999).

[18] T. Kunimoto, K. Nagasaka, H. Nojiri, S. Luther, M. Motokawa, H. Ohta, T. Goto, S. Okubo, and K. Kohn, Submillimeter Wave ESR Study of Magnetic Excitations in the Ising Ferromagnetic Chain $\mathrm{CoNb}_{2} \mathrm{O}_{6}$, J. Phys. Soc. Jpn. 68, 1703 (1999). 
[19] C. M. Morris, R. V. Aguilar, A. Ghosh, S. M. Koohpayeh, J. Krizan, R. J. Cava, O. Tchernyshyov, T. M. McQueen, and N. P. Armitage, Hierarchy of Bound States in the OneDimensional Ferromagnetic Ising Chain $\mathrm{CoNb}_{2} \mathrm{O}_{6}$ Investigated by High-Resolution Time-Domain Terahertz Spectroscopy, Phys. Rev. Lett. 112, 137403 (2014).

[20] R. Coldea, D. A. Tennant, E. M. Wheeler, E. Wawrzynska, D. Prabhakaran, M. Telling, K. Habicht, P. Smeibidl, and K. Kiefer, Quantum Criticality in an Ising Chain: Experimental Evidence for Emergent $E_{8}$ Symmetry, Science 327, 177 (2010).

[21] S. Sachdev and A. P. Young, Low Temperature Relaxational Dynamics of the Ising Chain in a Transverse Field, Phys. Rev. Lett. 78, 2220 (1997).

[22] M. Suzuki, Relationship Between d-Dimensional Quantum Spin Systems and $(d+1)$-Dimensional Ising Systems, Prog. Theor. Phys. 56, 1454 (1976).

[23] J. Zhang, F. M. Cucchietti, C. M. Chandrashekar, M. Laforest, C. A. Ryan, M. Ditty, A. Hubbard, J. K. Gamble, and R. Laflamme, Direct Observation of Quantum Criticality in Ising Spin Chains, Phys. Rev. A 79, 012305 (2009).

[24] J. J. Binney, N. J. Dowrick, A. J. Fisher, and M. E. J. Newman, The Theory of Critical Phenomena (Oxford University Press, New York, 1992).

[25] B. K. Chakrabarti, A. Dutta, and P. Sen, Quantum Ising Phases in Transverse Ising Models (Springer-Verlag, Berlin, Heidelberg, 1996).

[26] E. Husson, Y. Repelin, N. Q. Dao, and H. Brusset, Characterization of Different Bondings in Some Divalent Metal Niobates of Columbite Structure, Mater. Res. Bull. 12, 1199 (1977).

[27] K. Momma and F. Izumi, VESTA 3 for Three-Dimensional Visualization of Crystal, Volumetric and Morphology Data, J. Appl. Crystallogr. 44, 1272 (2011).

[28] E. M. da Silva Wheeler, Neutron Scattering from Low-Dimensional Quantum Magnets, Ph.D. thesis, Oxford University, 2007.

[29] S. Lee, R. K. Kaul, and L. Balents, Interplay of Quantum Criticality and Geometric Frustration in Columbite, Nat. Phys. 6, 702 (2010).

[30] T. Imai, C. P. Slichter, K. Yoshimura, and K. Kosuge, Low Frequency Spin Dynamics in Undoped and Sr-Doped $\mathrm{La}_{2} \mathrm{CuO}_{4}$, Phys. Rev. Lett. 70, 1002 (1993).

[31] T. Imai, C. P. Slichter, K. Yoshimura, M. Katoh, and K. Kosuge, Spin-Spin Correlation in the Quantum Critical Regime of $\mathrm{La}_{2} \mathrm{CuO}_{4}$, Phys. Rev. Lett. 71, 1254 (1993).
[32] T. Imai, C. P. Slichter, K. Yoshimura, M. Katoh, and K. Kosuge, High Temperature ${ }^{63,65} \mathrm{Cu} N Q R$ and NMR Study of the High Temperature Superconductor $\mathrm{La}_{2-x} \mathrm{Sr}_{x} \mathrm{CuO}_{4}$ (0 $\leq x \leq 0.15)$, Physica (Amsterdam) 197B, 601 (1994).

[33] S. Mukhopadhyay, M. Klanjsek, M. S. Grbic, R. Blinder, H. Mayaffre, C. Berthier, M. Horvatic, M. A. Continentino, A. Paduan-Filho, B. Chiari et al., Quantum-Critical Spin Dynamics in Quasi-One-Dimensional Antiferromagnets, Phys. Rev. Lett. 109, 177206 (2012).

[34] O. Vyaselev, M. Takigawa, A. Vasiliev, A. Oosawa, and H. Tanaka, Field-Induced Magnetic Order and Simultaneous Lattice Deformation in $\mathrm{TlCuCl}_{3}$, Phys. Rev. Lett. 92, 207202 (2004).

[35] H. Kuhne, A. A. Zvyagin, M. Gunther, A. P. Reyes, P. L. Kuhns, M. M. Turnbull, C. P. Landee, and H.-H. Klauss, Dynamics of a Heisenberg Spin Chain in the Quantum Critical Regime: NMR Experiment versus Effective Field Theory, Phys. Rev. B 83, 100407(R) (2011).

[36] F. L. Ning, K. Ahilan, T. Imai, A. S. Sefat, M. A. McGuire, B. C. Sales, D. Mandrus, P. Cheng, B. Shen, and H.-H. Wen, Contrasting Spin Dynamics between Underdoped and Overdoped $\mathrm{Ba}_{2}\left(\mathrm{Fe}_{1-x} \mathrm{Co}_{x}\right)_{2} \mathrm{As}_{2}$, Phys. Rev. Lett. 104, 037001 (2010).

[37] Y. Nakai, T. Iye, S. Kitagawa, K. Ishida, H. Ikeda, S. Kasahara, H. Shishido, T. Shibauchi, Y. Matsuda, and T. Terashima, Unconventional Superconductivity and Antiferromagnetic Quantum Critical Behavior in the IsovalentDoped $\mathrm{Ba}_{2} \mathrm{Fe}_{2}\left(\mathrm{As}_{1-x} \mathrm{P}_{x}\right)_{2}$, Phys. Rev. Lett. 105, 107003 (2010).

[38] R. Zhou, Z. Li, J. Yang, D. L. Sun, C. T. Lin, and G. q. Zheng, Quantum Criticality in Electron-Doped $\mathrm{Ba}_{2} \mathrm{Fe}_{2-x} \mathrm{Ni}_{x} \mathrm{As}_{2}$, Nat. Commun. 4, 2265 (2013).

[39] A. Narath, Nuclear Spin-Lattice Relaxation in Hexagonal Transition Metals: Titanium, Phys. Rev. 162, 320 (1967).

[40] J. Wu, M. Kormos, and Q. Si, Finite Temperature Spin Dynamics in a Perturbed Quantum Critical Ising Chain with an $E_{8}$ Symmetry, arXiv:1403.7222.

[41] S. Sachdev, T. Senthil, and R. Shankar, Finite-Temperature Properties of Quantum Antiferromagnets in a Uniform Magnetic Field in One and Two Dimensions, Phys. Rev. B 50, 258 (1994).

[42] V. Jaccarino, Proceedings of the International School of Physics, Enrico Fermi, Vol. XXXVII (Academic Press, New York, 1967), p. 335. 